\title{
Editorial:
}

\section{Advances in cardiovascular disease: how far is the summit?}

\author{
Jian-an WANG \\ Guest-Chief Editor, \\ Chairman of Qianjiang International Cardiovascular Congress \\ (Cardiovascular Key Lab of Zhejiang Province, the Second Affiliated Hos- \\ pital, School of Medicine, Zhejiang University, Hangzhou 310009, China) \\ E-mail: wang_jian_an@tom.com
}

doi:10.1631/jzus.B1001015

In the last three decades, great achievements have been made in the field of cardiovascular medicine. The rapid development of new drugs, the invention of novel devices and techniques, all of these, have significantly decreased the mortality and morbidity from cardiovascular disease. Mortality and morbidity rates fell by at least 50\% from about 1980 to 2000. However, are we winning the war against cardiovascular disease?

Cardiovascular diseases are still the major leading cause of global mortality and morbidity. As estimated by the World Health Organization, twenty million people will die from cardiovascular disease by 2015. Coronary artery disease (CAD) is especially increasing rapidly. In China, the incidence is increasing with the changing lifestyle and economic development, and affected by factors such as urbanization, decreased physical activity, increased tobacco use, and dietary changes. More than one million patients die from CAD every year in China. Atrial fibrillation is the most common arrhythmia, which increases the risk of stroke five-fold. Atrial fibrillation will become the new epidemic cardiovascular disease in the 21st century, as estimated by the World Heart Federation. Therefore, prevention of cardiovascular disease has become a key public health issue worldwide.

Although therapeutic options for cardiovascular disease are developing rapidly, not all patients receive the standard treatment. For example, changes in CAD treatment have been revolutionary. Percutaneous coronary intervention (PCI) has been increasingly used, and even with more complex lesions. In China, PCI use has increased by $30 \%$ to $40 \%$ in the last five years. However, many treatments are not applied in a standardized fashion, or according to guidelines, which increases medical risks, and may lead to the waste of medical resources. Standardization and adherence to guidelines will be an ongoing challenge.

In the last decade, a number of novel devices and

C Zhejiang University and Springer-Verlag Berlin Heidelberg 2010 technologies have been developed in cardiovascular medicine. Cardiac cell therapy has caused great excitement. Both clinical trial and basic research have provided evidence that transplantation of stem cells can improve cardiac function through a paracrine effect, angiogenesis, and possibly myogenesis. However, there are still many substantial obstacles and setbacks along the road of successful application of this therapy. For instance, the majority of implanted cells die within one week of transplantation. This and other technical problems will require further research before this therapy is readily applied.

Motivated by these issues, we will hold the 4th Qianjiang International Cardiovascular Congress (QICC4) and Annual Scientific Session of the Zhejiang Society of Cardiology in Hangzhou from July 29 to August 1, 2010. The theme for this congress is "Focus on Prevention, Comply with Guidelines, Start from the Beginning, Go to the Summit”.

QICC4 will deliver, in an independent forum, up-to-date achievements in new theories, technologies, and guidelines applied to this field, including general cardiology, interventional cardiology, electrophysiology, and basic research. Also, knowledge and skill contests, live case presentations, presentations on difficult cases, and late breaking clinical trials will be popular sessions.

Of note, the Hong Kong College of Cardiology (HKCC) and the Taiwan Society of Cardiovascular Interventions (TSCI) are the supporters of QICC4, where a number of overseas academic experts will give lecture and live case demonstrations.

In this special issue of QICC, we received many papers from experts at home and abroad, the contents of which include the current status of PCI, stent restenosis, hypertension, pacing technology, case reports of stent implantation, stem cell research, and many other topics of interest.

On behalf of the organizing committee and Zhejiang Society of Cardiology, I am honored to invite your participation in this meeting and look forward to seeing you at QICC4 this summer.

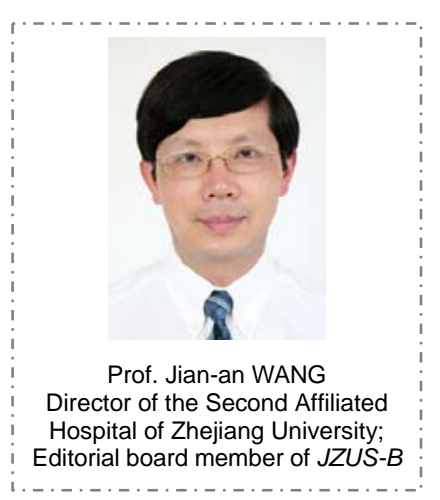

